\title{
Electron Reconstruction and Identification with the ATLAS Detector
}

\author{
O. Arnaez on behalf of the ATLAS collaboration \\ LAPP, Université de Savoie, CNRS/IN2P3, Annecy-le-Vieux, France
}

\begin{abstract}
Several Standard Model processes contain electrons in the final state. Also, many signatures expected from physics beyond the Standard Model include one or more electrons. This paper describes the electron reconstruction along with some of the identification criteria used in the ATLAS detector. The expected performance at the start-up of the experiment are also presented.
\end{abstract}

Keywords: ATLAS, LHC, electron, reconstruction, identification, track, shower, electromagnetic PACS: $01.30 . \mathrm{Cc}, 29.40 . \mathrm{Vj}, 29.40 . \mathrm{Gx}$

\section{INTRODUCTION}

The LHC nominal center-of-mass energy being $14 \mathrm{TeV}$, a wide variety of known physics processes $-\mathrm{J} / \psi, \mathrm{Y}, \mathrm{W} / \mathrm{Z}$, heavy flavour decays- and potentially new physics processes will produce electrons in a wide energy range from a few $\mathrm{GeV}$ up to a few $\mathrm{TeV}$. At the nominal luminosity $\left(10^{34} \mathrm{~cm}^{-2} \mathrm{~s}^{-1}\right)$ the inelastic event rate will be about 600 million per second. Thus it is expected to see a huge number of jets and pile-up events faking electrons. Hence, it is important to have a robust method for the reconstruction and efficient criteria for the identification of the electrons.

\section{THE ELECTRON RECONSTRUCTION}

The reconstruction of the electrons in ATLAS relies on the signal produced by two subdetectors : the inner detector tracker and the electromagnetic calorimeter[1].

The former is composed of a pixel detector, a silicon microstrip (SCT) and a transition radiation (TRT) trackers enclosed in a $2 \mathrm{~T}$ magnetic field. The reconstruction of the tracks covers a large precision region in pseudo-rapidity, up to $\eta<2.5$. The latter is a $\mathrm{Pb} / \mathrm{LAr}$ calorimeter with accordion geometry segmented in three layers ("strips", "middle" and "back") allowing to measure the longitudinal extension of the electromagnetic shower, preceded by a thin presampler to estimate the energy loss in the upstream material.

The reconstruction of electrons usually starts by the independent reconstructions of tracks from the hits in the inner detector and electromagnetic clusters from the cells in the calorimeter.

The tracks reconstruction involves the inside-out algorithm starting from seeds in the pixel/SCT layers and finding hits in the TRT and the back-tracking algorithm starting from hits in the TRT and extrapolating them to the SCT and the pixel layers. Both algorithms use track fitters taking into account the fraction of energy radiated in the inner detector by bremsstrahlung process. 
The reconstruction of electromagnetic clusters uses a sliding-window algorithm. It consists of mapping the calorimeter cells into $\eta=0.025$ towers and in moving a window of $5 \times 5$ towers on this grid. A cluster seed is found when the transverse energy in the window is greater than $3 \mathrm{GeV}$. A cluster with a size corresponding to $3 \times 7$ cells in the middle layer is then formed and its position is refined. Different corrections are applied to the cluster position and energy in order to take into account the variation with the particle impact position (S-shape and energy containment along $\eta$ and accordion geometry effect along $\phi$ ). Also, in the transition region between the barrel and end-cap parts of the calorimeter $(1.37<\eta<1.52)$, a specific correction is applied. Finally, the calibration between the different calorimeter layers leads to the application of longitudinal weights accounting for the energy scale $(\lambda)$, loss of energy in the upstream/dead material ( $\mathrm{m}$ and $\left.W_{0}\right)$ and the longitudinal leakage $\left(W_{3}\right)$ as follows :

$$
E_{\text {rec }}=\lambda\left(m+W_{0} E_{P S}+E_{\text {strips }}+E_{\text {middle }}+W_{3} E_{\text {back }}\right)
$$

These coefficients are $\eta$-dependent (following the upstream material distribution) and are precisely determined from the full simulation of the detector. Such a reconstruction mechanism gives a linearity of the calorimeter response which is better than $1 \%$ over the 20-500 GeV energy range with the energy resolution depicted in Figure 1.

After their extrapolation to the strips and middle samplings of the calorimeter, tracks are matched to the cluster using $\left|\Delta \eta_{\text {trk } / \text { strips }}\right|<0.05$ and $-0.1<\operatorname{sign}(q) \Delta \phi_{\text {trk } / \text { middle }}<0.05$, the asymmetry accounting for the bremsstrahlung. In a busy environment where multiple tracks can be associated to one cluster, the association is done with the track giving the ratio $E_{\text {cluster }} / p_{\text {trk }}$ closest to 1 .
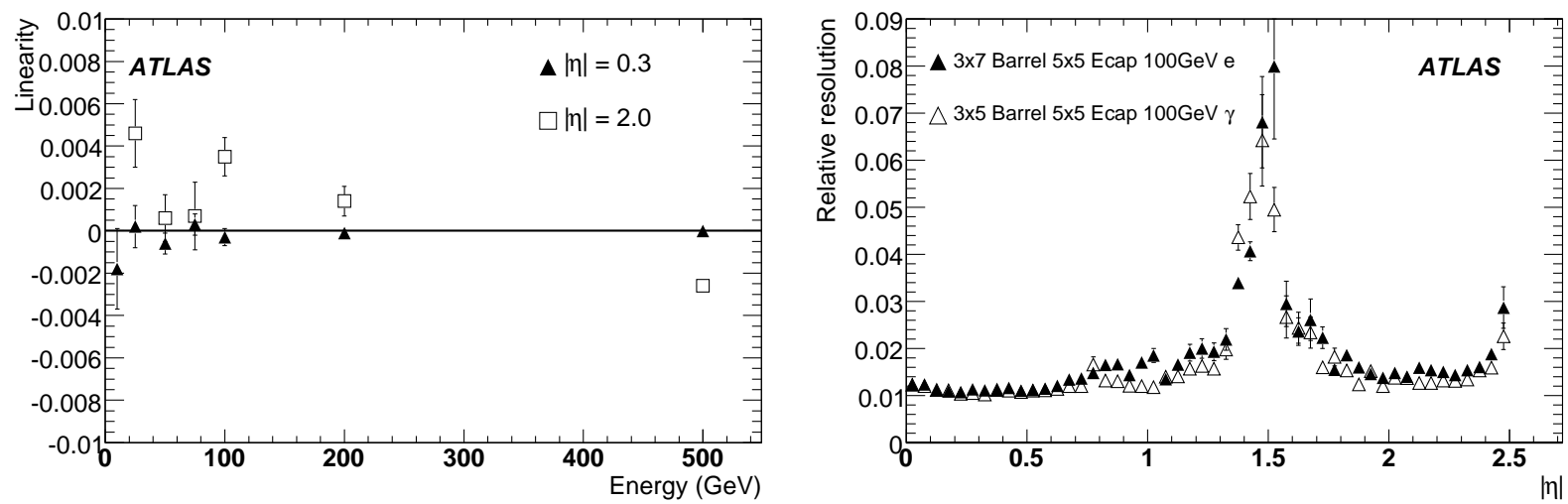

FIGURE 1. Left: Linearity of the energy response of the calorimeter vs energy of the incoming electron. Right: Relative energy resolution vs pseudorapidity $\eta$ for $100 \mathrm{GeV}$ electrons (black).

\section{ELECTRON IDENTIFICATION}

At the LHC, QCD processes will dominate and jets -as they produce tracks and deposit energy in the electromagnetic calorimeter- will fake electrons. At this stage, the true electron fraction (purity) is expected to be $O\left(10^{-5}\right)$. Variables reflecting the shower shape in the calorimeter, the track quality, tight constraints on the track-cluster matching 
and identification techniques using the TRT capabilities are needed to separate the true electrons from the fakes.

The electron and jet showers exhibit significant differences. Using the segmentation and the thin granularity of the calorimeter, jets will be seen broader (see Figure 2, left) and have more leakage in the hadronic calorimeter than electrons.
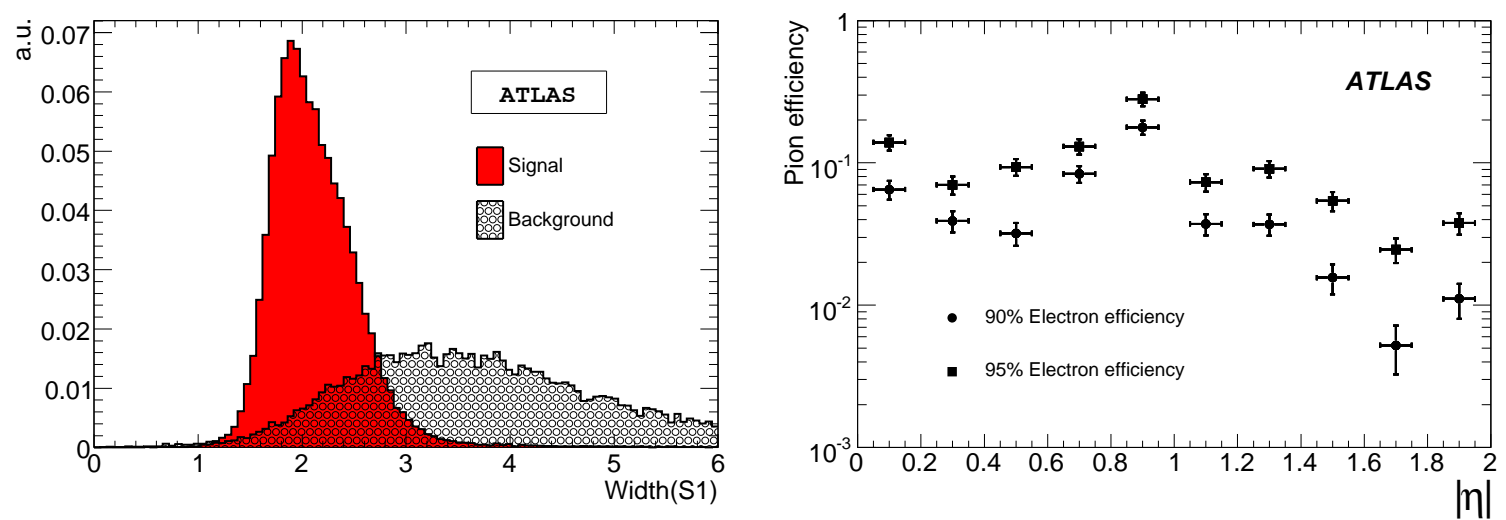

FIGURE 2. Left: Distributions representing the shower width in the strip layer for the signal electrons (red) and the QCD fakes background (black). Right: Pion efficiency vs $\eta$ for $90 \%$ and $95 \%$ efficiency on $25 \mathrm{GeV}$ electrons.

With the ratio of high-threshold hits and the time-over-threshold, the TRT also provides capabilities to separate pions and electrons as shown in Figure 2 (right).

Finally, the quality of the track (number of hits in the pixel, SCT and TRT layers) and of the track-cluster association can be used to further reduce the background.

Based on these variables, different sets of cuts -"Loose", "Medium" and "Tight"- used to identify electrons are defined with gradual rejection power. The expected efficiencies for electrons for the different sets and the level of expected contamination by fakes after the Tight cuts are shown in Figure 3. A rejection factor of $10^{5}$ is achieved and for $E_{T}>30$ $\mathrm{GeV}$, the signal-over-background ratio for isolated electron is about 1 .

\section{EFFICIENCY MEASUREMENTS}

It is important to measure the electron efficiencies from data in order not to rely on MC predictions for the detector response. The reconstruction and identification efficiencies can be measured using the so-called "tag-and-probe" method with $Z \rightarrow e e$ events.

The idea is to select electron pairs with invariant mass close to $M_{Z}$ and tight cuts on the first electron (called tag electron) in order to have high signal-to-background ratio in the sample. The efficiency of various cuts can be measured on the second electron (called probe electron). The ratio of probes passing the cuts over the total number of probes considered directly provides the efficiency of the tested cuts.

Figure 4 shows a good agreement between identification efficiencies obtained from the Monte Carlo truth and the ones measured using tag-and-probe. 

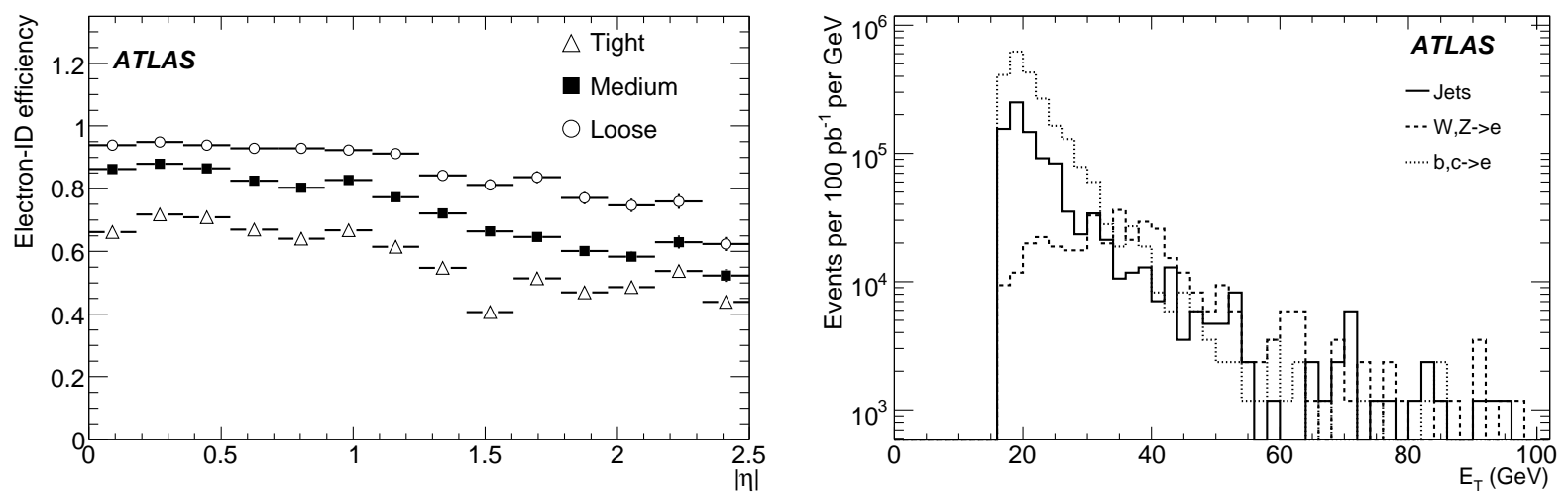

FIGURE 3. Left: Electron identification efficiency as a function of eta for electrons with $E_{T}>5 \mathrm{GeV}$ from $H \rightarrow$ eeee decays. Right: Differential cross-sections as a function of $E_{T}$ after tight cuts, shown separately for various sources of isolated electrons, non-isolated electrons and residual jet background, for an integrated luminosity of $100 \mathrm{pb}^{-1}$ and for the simulated with $E_{T}>17 \mathrm{GeV}$ di-jet sample.
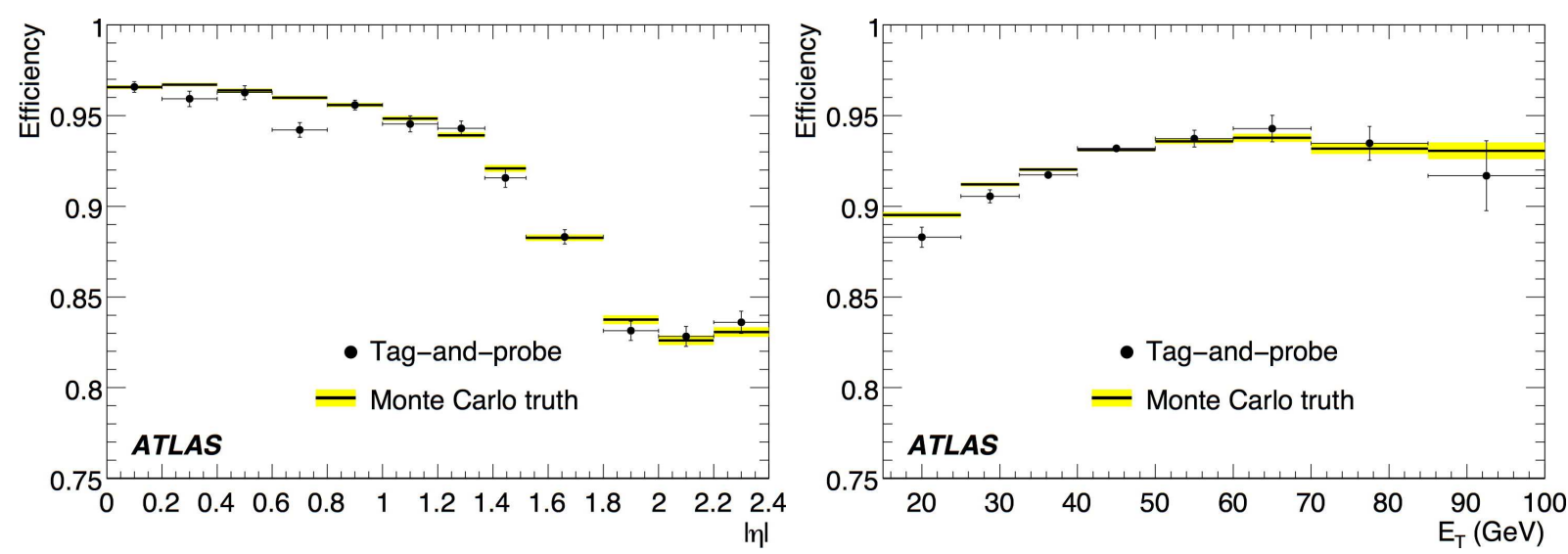

FIGURE 4. Efficiency of the electron reconstruction as a function of $|\eta|$ (left) and $E_{T}$ (right) for $Z \rightarrow e e$ decays, using the tag-and-probe method and the Monte Carlo truth information. Errors are statistical and correspond to an integrated luminosity of $100 \mathrm{pb}^{-1}$.

\section{CONCLUSION}

Good performance can be achieved with the ATLAS detector using robust reconstruction and identification methods. First collisions will soon be used to measure efficiencies, to improve the performance and to tune the Monte Carlo before using electrons to perform precision measurements and (re-)discoveries.

\section{REFERENCES}

1. The ATLAS Collaboration, Expected Performance of the ATLAS Experiment - Detector, Trigger and Physics, arXiv:0901.0512, 2009 